Artículo original

Recibido para publicación: 10 de octubre de 2015 Aceptado para publicación: 18 de noviembre de 2015

\title{
DOBLE PERIODO DE VACACIONES COMO DERECHO DE CARÁCTER PRINCIPAL A TRABAJADORES CATALOGADOS DE ALTO RIESGO
}

\section{Double holiday period as main character right workers cataloged high risk}

\author{
Autores: \\ José Fabián Roca Franco'. \\ Jhoen Albany Escobar Alcaraz². \\ Correspondencia en: jose.roca@curvirtual.edu.cojescobara13@curnvirtual.edu.co
}

\begin{abstract}
RESUMEN
El presente artículo de reflexión tiene como finalidad realizar proposiciones en torno a la extensión del beneficio de doble periodo de vacaciones para otro tipo de trabajadores diferentes de los señalados en el artículo 186 Núm. 2 del vigente Código Sustantivo de Trabajo, teniendo en cuenta que existen otras circunstancias de alto riesgo que ameritan ser consideradas para otorgar esta prerrogativa a otro tipo de ocupaciones, profesiones u oficios. .
\end{abstract}

Este artículo se deriva de una investigación de carácter descriptivo, de contenido crítico normativo, con un método deductivo.

\section{Palabras claves}

Doble periodo vacaciones, trabajadores de alto riesgo, beneficio legal, derecho principal.

\begin{abstract}
This article aims reflection make proposals concerning the extension of the benefit of double holiday period for different workers other than those specified in Article 186 no. 2 current Labour Code, given that there other high-risk circumstances that merit consideration for granting this privilege to other occupations, professions and trades. .

This article is derived from an investigation descriptive, normative critical content, with a deductive method.

\section{Keywords}

Double holiday period, workers in high-risk, legal benefit, main right.

\footnotetext{
${ }_{1}^{1}$ Abogado. Especialista en Seguridad Social. Docente programa de Derecho de la Corporación Universitaria Rafael Núñez.

2 Estudiante del programa de Derecho de la Corporación Universitaria Rafael Núñez, noveno semestree nocturno.
} 


\section{INTRODUCCIÓN}

Desde inicios del siglo $\mathrm{XX}$, el descanso remunerado comienza a tener sus cimientos legales dentro de las legislaciones del mundo, luego de diversas luchas socio políticas por alcanzarlo. La OIT en 1919 inicia su accionar en pos de los trabajadores forjando las directrices del trabajo, y fue en el año de 1936 cuando el socialista francés, León Blum, impulsa una serie de reformas en aspectos de política laboral, que procuraban un incremento salarial, seguridad social y vacaciones remuneradas. Se tiene, que "El primero de junio de 1936 el parlamento acuerda la reducción de la jornada fijándola en 40 horas semanales y el 21, el derecho a tener 15 días de vacaciones pagadas al año...". Posteriormente, en el año de 1948, con la Declaración Universal de Derechos Humanos, se ratifica en su artículo 24, el periodo de vacaciones como un derecho laboral fundamental, cuando expresa que "Toda persona tiene derecho al descanso, al disfrute del tiempo libre, a una limitación razonable de la duración del trabajo y a vacaciones periódicas pagadas".

A raíz de lo anterior, en Colombia, escenario de multiplicidad de contrastes en materia laboral, comienza como sociedad encausada por el reconocimiento de los derechos humanos, en el año de 1963 a ratificar en materia de descanso remunerado el Convenio 52 de la Organización Internacional del Trabajo (OIT). Luego, en el año de 1950 con el Decreto Ley 2663 en su artículo 188, correspondiente al artículo 186 del Decreto Ley 3743 del año indicado (Código Sustantivo del Trabajo) define lo concerniente al periodo de descanso remunerado para los trabajadores Colombianos. Más tarde, con la entrada en vigencia de la Constitución de 1991, los principios rectores que rigen para el trabajo y empleados colombianos, sumado al bloque de constitucionalidad, constituyen el pilar normativo para este aspecto tan importante. 
Pero aún se puede apreciar diferencias en la aplicación de normas, y que están directamente relacionadas con derechos fundamentales del trabajador, tal es el caso de lo que ocurre con los profesionales y ayudantes que trabajan en establecimientos privados dedicados a la lucha contra la tuberculosis, y los ocupados en la aplicación de rayos $\mathrm{X}$, quienes tienen derecho a gozar de quince (15) días de vacaciones remuneradas por cada seis (6) meses de servicios prestados. Situación que debería ser considerada por el legislador, habida cuenta que existen en el país, gran número de personas que se exponen constantemente ante agentes químicos o situaciones de alto riesgo en sus sitios de labores, semejantes a las descritas en el Código Sustantivo del Trabajo en su artículo 186 Núm. 2. Lo descrito, tendría mayor soporte, si se tiene en cuenta que ya en Colombia se han tenido presente este tipo de escenarios para materializar beneficios legales, verbi gracia, en el campo de pensiones con lo dispuesto en el artículo $4^{\circ}$ del Decreto 2090 de 2003 que indica que gozan de beneficios pensiónales (menor edad y/o menor semana de cotización), pudiendo ser extensivo a ellos el doble periodo de vacaciones en un año, en razón al ejercicio de su actividad.

Así las cosas, sea este el momento para instar dentro del escenario normativo que rigen la materia, para que el debate sobre este tipo de situaciones procure una perspectiva y posterior a ello acciones contundentes para que a todos aquellos trabajadores colombianos que afrontan circunstancias semejantes a las contempladas en el artículo 186 Núm. 2 del vigente Código Sustantivo de Trabajo, se les reconozca este importante beneficio. 


\section{MÉTODOS.}

El presente artículo de reflexión es de corte descriptivo - propositivo, orientado bajo un método deductivo, fue desarrollado a partir de fuentes bibliográficas relacionadas con el derecho de las vacaciones en materia laboral.

\section{RESULTADOS Y DISCUSIÓN.}

\section{Periodo de descanso remunerado un derecho principal desde principios del siglo $\mathrm{XX}$}

Vacación, o como es utilizada en todo el orbe de manera plural, vacaciones, etimológicamente, viene del latín vocatio, vacatîonis, que significa tiempo en que un cargo estaba vacante o desocupado, o descanso temporal de una actividad laboral o habitual, o como lo indica la Real Academia Española "Tiempo que dura la cesación de un trabajo" "Acción de vacar (quedar un empleo o cargo sin persona que lo desempeñe)" ( Real Academia Española, DRAE, 2014)

En materia de derecho laboral, la historia devela que, las primeras acciones en pos de legalizar el periodo de vacaciones, se dio en el año de 1936, cuando el socialista francés, León Blum, impulsa una serie de reformas en aspectos de política laboral, que procuraban un incremento salarial, seguridad social y vacaciones remuneradas. Se tiene, que "El primero de junio de 1936 el parlamento acuerda la reducción de la jornada fijándola en 40 horas semanales y el 21, el derecho a tener 15 días de vacaciones pagadas al año. Para facilitar los desplazamientos, Leo Lagrange (nombrado subsecretario de Estado para los deportes y el tiempo libre) negocia con la compañía de ferrocarriles el establecimiento de un "billete de vacaciones anuales" con un $40 \%$ de reducción. 
En 1936 seiscientos mil (600.000) obreros se marchan de vacaciones; al año siguiente son un millón ochocientos mil." (Lóyzaga de la Cueva, 2012). Lo anterior fue el primer precedente histórico de migración masiva de obreros a lugares diferentes de sus sitios de trabajo, con el propósito de dar cumplimiento a la necesidad humana de descansar de la rutina.

Tal fue y sigue siendo su relevancia que la Organización Internacional del Trabajo (OIT), creada luego de la devastadora primera guerra mundial, y posteriormente la primera agencia de las Naciones Unidas. En su misión por promover, fomentar y mejorar los derechos, oportunidades y protección de las condiciones de los trabajadores, en pos de sus actividades como organismo internacional, estableció la prevalencia del periodo de descanso para las personas como un derecho de carácter principal, y que debe ser remunerado. Así quedó establecido en el Convenio 52 de 1936 en su artículo segundo (2):

"1. Toda persona a la que se aplique el presente Convenio tendrá derecho, después de un año de servicio continuo, a unas vacaciones anuales pagadas de seis días laborables, por lo menos.

2. Las personas menores de dieciséis años, incluidos los aprendices, tendrán derecho, después de un año de servicio continuo, a vacaciones anuales pagadas de doce días laborables, por lo menos.

3. No se computan a los efectos de las vacaciones anuales pagadas:

(a) los días feriados oficiales o establecidos por la costumbre;

(b) las interrupciones en la asistencia al trabajo debidas a enfermedad.

4. La legislación nacional podrá autorizar, a título excepcional, el fraccionamiento de la parte de las vacaciones anuales que exceda de la duración mínima prevista por el presente artículo.

5. La duración de las vacaciones anuales pagadas deberá aumentar progresivamente con la duración del servicio, en la forma que determine la 
legislación nacional.. (OIT. C052 - Convenio sobre las vacaciones pagadas, 1936, Núm. 52)

Posteriormente, en el año de 1948, con la Declaración Universal de Derechos Humanos, se ratifica en su artículo 24, el periodo de vacaciones como un derecho laboral fundamental, cuando expresa que "Toda persona tiene derecho al descanso, al disfrute del tiempo libre, a una limitación razonable de la duración del trabajo y a vacaciones periódicas pagadas". Es desde este momento, en donde concepciones erróneas en cuanto a este concepto, como la cosmovisión de muchos sectores, sobre todo los dueños de los medios de producción, quienes afirmaban que este descanso extenso era sinónimo de pereza, pierde piso y se posiciona la aceptación general, que obedece a la realidad biológica, la cual indica que el cuerpo requiere recuperación de la energía que gasta durante el desarrollo de rutinas extensas de trabajo. Este punto se puede destacar observando el proceso posterior, el cual consolida este periodo anual como un derecho fundamental en la mayoría de legislaciones a lo largo y ancho del mundo entero.

\section{Periodo descanso remunerado en Colombia}

Colombia, en este sentido, y de conformidad a lo que expresa el artículo 93 Superior "Los tratados y convenios internacionales ratificados por el Congreso, que reconocen los derechos humanos y que prohíben su limitación en los estados de excepción, prevalecen en el orden interno. Los derechos y deberes consagrados en esta Carta, se interpretarán de conformidad con los tratados internacionales sobre derechos humanos ratificados por Colombia". (Henao Hadrón, 2010: 60). Llegado a este punto, hay que advertir que en aras de que sea armonizado el principio de supremacía de la Constitución, del cual data el artículo 4 , con el de prevalencia de los tratados de derechos humanos, ratificados por el 
Estado colombiano, conforman en sí, el llamado Bloque de Constitucionalidad, el cual significa que las normas consagradas en estos convenios, se ubican en el nivel de la norma de normas, imperando al igual que el resto del articulado Constitucional sobre la misma ley. Por tal motivo la Constitución Política de 1991 erige como uno de los derechos fundamentales y principios rectores el trabajo, de donde se desprenden garantías prestacionales e irrenunciables como es el caso de las vacaciones, las cuales pueden disfrutar bajo la protección Constitucional y legal todo trabajador colombiano, así lo ha confirmado la Sala Plena de la H. Corte Constitucional cuando haciendo referencia al descanso enuncia:

"uno de los derechos fundamentales del trabajador, es el descanso, el cual está definido por el Diccionario de la Real Academia como quietud o pausa en el trabajo o fatiga"[6]. En efecto, la ius fundamentalidad de este derecho se deduce de la interpretación sistemática [7] de los artículos 1ㅇ, 25 y 53 de la Carta, en tanto y cuanto el descanso es una consecuencia necesaria de la relación laboral y constituye uno de los principios mínimos fundamentales del trabajo" ( corte constitucional, Sentencia T-076 del 2001)

Por lo que sigue, el artículo 53 de la Constitución Política, consagra dentro de los principios rectores para el estatuto del trabajo en Colombia, entre otros, la "...primacía de la realidad sobre formalidades establecidas por los sujetos de las relaciones laborales; garantía a la seguridad social, la capacitación, el adiestramiento y el descanso necesario...". Además, confirma lo anteriormente expuesto cuando establece en su inciso cuarto que "Los convenios internacionales del trabajo debidamente ratificados hacen parte de la legislación interna" (Henao Hadrón, 2010: 38). Sin duda alguna, en nuestro ordenamiento jurídico interno las vacaciones, son un derecho fundamental que 
dentro de la misma relación, es irrenunciable, por lo cual tiene en el marco normativo carácter de obligatoriedad.

Ahora bien, el Código Sustantivo del Trabajo, en su artículo 186, expresa:

"1. Los trabajadores que hubieren prestado sus servicios durante un (1) año tienen derecho a quince (15) días hábiles consecutivos de vacaciones remuneradas". (Decreto-Ley 2663 de 1950)

El anterior aspecto en la praxis obra como regla general, pero en tratándose de algunos trabajadores que laboran en el ámbito de la salud, el legislador ha determinado quince (15) días hábiles de vacaciones cada seis (6) meses, lo cual está consagrado en la misma norma referenciada cuando dice:

"2. Los profesionales y ayudantes que trabajan en establecimientos privados dedicados a la lucha contra la tuberculosis, y los ocupados en la aplicación de rayos $X$, tienen derecho a gozar de quince (15) días de vacaciones remuneradas por cada seis (6) meses de servicios prestados" ( Decreto-Ley 2663 de 1950)

\section{Doble periodo de vacaciones como derecho principal para trabajadores catalogados de alto riego}

En suma, las vacaciones para personas profesionales o ayudantes de establecimientos privados que trabajan bajo condiciones de exposición a riesgos desencadenados por los diferentes procedimientos dentro del contexto de 
enfermedades como la tuberculosis y que en sus actividades ejecutadas en el sitio de trabajo tienen que llevar a cabo ocupaciones con la aplicación de rayos $\mathrm{X}$, bien tienen en la legislación colombiana un tratamiento jurídico especial, toda vez que los peligros son inminentes. De acuerdo con lo anterior, y si echamos un vistazo sobre este aspecto, vemos como la norma alude a estos escenarios para que se active o se ejercite el derecho. Sin embargo, también a menudo se denotan en el entorno laboral altos riesgos que exponen seriamente al trabajador, en cuanto a su desgaste psicofísico. Por lo tanto, no pueden descartarse de tajo estas determinadas circunstancias, pues surgen con ocasión del trabajo.

Tomando como referencia el tratamiento especial de los profesionales y ayudantes que laboran en establecimientos privados contra la lucha de la tuberculosis y los ocupados en la aplicación de rayos $\mathrm{X}$, encontramos en el ordenamiento jurídico, de modo paralelo, una clase de trabajadores cuya actividad se le ha catalogado de alto riesgo, contenido en el Decreto 2090 de 2003, los cuales gozan de beneficios pensiónales (menor edad y/o menor semana de cotización), pudiendo ser extensivo a ellos el doble periodo de vacaciones en un año, en razón al ejercicio de su actividad. Señala el artículo $2^{\circ}$ del Decreto 2090 de 2003:

"Actividades de alto riesgo para la salud del trabajador. Se consideran actividades de alto riesgo para la salud de los trabajadores las siguientes:

1. Trabajos en minería que impliquen prestar el servicio en socavones o en subterráneos.

2. Trabajos que impliquen la exposición a altas temperaturas, por encima de los valores límites permisibles, determinados por las normas técnicas de salud ocupacional. 
3. Trabajos con exposición a radiaciones ionizantes.

4. Trabajos con exposición a sustancias comprobadamente cancerígenas.

5. En la Unidad Administrativa Especial de Aeronáutica Civil o la entidad que haga sus veces, la actividad de los técnicos aeronáuticos con funciones de controladores de tránsito aéreo, con licencia expedida o reconocida por la Oficina de Registro de la Unidad Administrativa Especial de Aeronáutica Civil, de conformidad con las normas vigentes"(Decreto 2090 de 2003).

6. En los Cuerpos de Bomberos, la actividad específica de actuar en operaciones de extinción de incendios.

En el Instituto Nacional Penitenciario y Carcelario, Inpec, la actividad del personal dedicado a la custodia y vigilancia de los internos en los centros de reclusión carcelaria, durante el tiempo en el que se ejecuten dicha labor. Así mismo, el personal que labore en las actividades antes señaladas en otros establecimientos carcelarios, con excepción de aquellos administrados por la fuerza pública.

Para gozar de quince días de vacaciones remuneradas por cada seis meses de trabajo, y el beneficio pensional de menor edad y/o menor semanas de cotización, se tiene en cuenta la actividad frente a los efectos nocivos de la salud, cabiendo la posibilidad de otorgar el derecho al doble periodo de vacaciones en un año a los trabajadores relacionados en los numerales $1^{\circ}, 2^{\circ}, 3^{\circ}$ y $4^{\circ}$ del artículo $2^{\circ}$ del Decreto 2090 de 2003, frente al Código Sustantivo del Trabajo.

A la luz de la nueva normatividad en materia de riesgos laborales, producto de los nuevos cambios y trasformaciones en el universo del trabajo, que se derivan de los avances o desarrollo tecnológico, científico, industrial, químico, entre otros, se hace necesario estudiar con sentido crítico el panorama que devela 
determinadas condiciones en las cuales se desarrollan diferentes tipos de actividades $\mathrm{u}$ ocupaciones que dejan expuestas al trabajador ante agentes, elementos o sustancias químicas. Siendo lo anterior, motivo esencial para invocar el derecho a un doble descanso anual o periodo de vacaciones por cada seis (6) meses, tal y como lo establece el artículo 186 numeral $2^{\circ}$ del Cogido Sustantivo del Trabajo. El Gobierno Nacional, en ejercicio de su deber estatal, previo concepto del Consejo Nacional de Riesgos Laborales, determina en forma constante las enfermedades que se consideran como laborales, apoyado por los Ministerios de Salud y Protección Social y del Trabajo, quienes en desarrollo de sus funciones les corresponde realizar una actualización de la tabla de enfermedades laborales, por lo menos cada tres (3) años. Lo anterior, atendiendo los estudios técnicos financiados por el Fondo Nacional de Riesgos Laborales. El Decreto 1477 del 2014, por el cual se expide la Tabla de Enfermedades Laborales., es precisamente el resultado vigente de esta labor. En el Anexo Técnico que lo integra, Sección II Parte A, hace alusión a enfermedades laborales directas como la Asbestosis, Silicosis, Neumoconiosis del minero de carbón y el Mesotelioma maligno, patologías que surgen por exposición a agentes químicos. Así mismo, en la Parte B, se señala en el Grupo II Cánceres de origen laboral.

Sin perder el contexto, parece perfectamente claro que como consecuencia de una exposición laboral en el nivel que describe el artículo 186 numeral ㄴo del CST, el cual trae como beneficio el poder ejercitar un periodo vacacional cada seis (6) meses, desata o da pie a la posibilidad de que los empleados que ostenten estas mismas condiciones de alto riesgo laboral, como las que data el Decreto 2090 de 2003, puedan hacerse merecedores al mismo derecho que a su vez es fundamental e irrenunciable, ya que el principio rector a la igualdad seria techo suficiente para que sea considerado por el legislador.

Cabe entonces preguntarse: 
¿Por qué razón lo consagrado en el Código Sustantivo del Trabajo en su artículo 186 numeral $2^{\circ}$ que dice "Los profesionales y ayudantes que trabajan en establecimientos privados dedicados a la lucha contra la tuberculosis, y los ocupados en la aplicación de rayos $X$, tienen derecho a gozar de quince (15) días de vacaciones remuneradas por cada seis (6) meses de servicios prestados", no puede ser aplicado a los demás trabajadores que a diario son expuestos en iguales o superiores niveles a actividades de alto riesgo laboral?.

A la postre, sólo sigue vigente y aplicándose a todos los demás empleados que se asemejen a este cuadro, el derecho básico o por regla general de quince (15) días hábiles consecutivos de vacaciones remuneradas, especificado en el numeral 1 del artículo 186 del CST.

Ante esta situación, la Honorable Corte Constitucional por vía jurisprudencial ante la acción de demanda de inconstitucionalidad, se ha ratificado en este derecho para los trabajadores dedicados a la lucha contra la tuberculosis o a la aplicación de rayos $X$, en el sentido que los beneficios bajo el principio de conservación del derecho, en este caso relacionados con el doble periodo de vacaciones anuales, no pueden ser excluidos de prestaciones económicas derivadas de la misma evolución normativa. Lo anterior fue objeto de estudio en la Sentencia C-669 del 2006 cuando la autoridad corporativa expresó:

“...No obstante, se observa que la expresión "por año cumplido" podría llevar a dos interpretaciones del artículo 1 de la Ley 995 de 2005: una primera, según la cual el aparte subrayado sólo cobijaría a quienes tienen período de causación anual, con lo cual quedarían excluidos aquéllos trabajadores cuyas vacaciones se causan semestralmente, los que, por tanto, no tendrían derecho a la compensación proporcional de sus 
vacaciones desde el momento de su vinculación. Una segunda, según la cual la norma demandada constituye apenas una regla general, redactada en términos de quienes ordinariamente causan sus vacaciones por años, que por tanto no excluye su aplicación a los trabajadores que tienen un sistema de causación semestral, pero que no por ello pierden su derecho a la compensación del descanso no disfrutado"(Corte Constitucional, Sentencia C- 669 de 2006)

Lo anterior, da pie para que en aras de que el derecho a un doble descanso anual sea aplicado a los demás trabajadores que se exponen a altos riesgos laborales, también sea en su momento objeto de análisis constitucional, pues de conformidad a lo expuesto, existen personas, empleadas bajo situaciones, reiteramos, de alto riesgo semejante o quizás superior a las cobijadas por el articulo 186 numeral $2^{\circ}$ del CST, no olvidando además, que esta norma es del año 1950, quedando desactualizada ante los avances de la ciencia.

Finalmente, la misma doctrina Nacional ha registrado en virtud de lo expuesto, que efectivamente es necesario se asuma esta medida o mejor dicho, se extienda este derecho a otros empleados que laboran bajo circunstancias de alta exposición a agentes químicos que pueden causar severos daños psicofísicos. Al respecto, y a manera de ejemplo, los doctores Fonseca, Heredia y Navarrete, médicos generales y cirujanos ante toda la población trabajadora expuesta existente a determinadas sustancias químicas de alto riesgo como los hidrocarburos aromáticos BTX dicen que se debe "... implementar el programa de vigilancia médica incorporando los aspectos clínicos y de diagnóstico de laboratorio para generar estrategias oportunas que conllevan al mantenimiento de adecuadas condiciones de salud de los trabajadores y que permitirá detectar las alteraciones producidas por los mismos en un estadio reversible; esta vigilancia médica debe ajustarse a las distintas necesidades y los recursos de las industrias 
en cuyos procesos se utilizan los hidrocarburos aromáticos. (Benceno, tolueno y xileno)" (Fonseca Patiño, 2010) Es de anotar que estos trabajadores, se exponen a diario a sustancias como el benceno, el tolueno, el ortoxileno, para-xileno, metaxileno y etil-benceno, entre otras, que tienen la propiedad de disolver cuerpos grasos, sobre todo del sistema nervioso central, periférico y de la medula ósea. Son componentes de artículos usados en la fabricación de pinturas, productos farmacéuticos, colas o adhesivos, desengrasantes, agentes limpiadores, en la producción de polímeros, plásticos, textiles, etc. Cabe señalar, que lo anterior exige del legislador un análisis profundo, habida cuenta que se trata de un derecho especial por vía de excepción para personas que por su trabajo, enfrentan de forma latente, riesgos inmensos.

\section{CONCLUSIONES}

Cabe concluir, que la aplicación a tiempo del derecho a un doble periodo de vacaciones anual, por extensión a los casos de los empleados expuestos a las sustancias químicas peligrosas, puede mejorar las condiciones psicofísicas de los trabajadores, evitar cambios biológicos negativos que van en detrimento de la salud, y permite un espacio de recuperación suficiente para regenerar las energías y el deterioro producto del constante riesgo asumido en el trabajo, so pena de que en algún momento sea irreversible. Además, en el estricto sentido jurídico, y luego de hacerse un estudio juicioso comparativo entre los casos señalados en el artículo 186 numeral $2^{\circ}$ y otros escenarios de alto riesgo laboral, como los referidos en este artículo, que se presentan en Colombia, por el principio rector y derecho fundamental Constitucional a la igualdad, así como sucede actualmente en materia de pensiones, es menester del legislador o en su defecto por vía jurisprudencial de la Corte Constitucional procurar se les sea otorgado este mismo derecho. 


\section{REFERENCIAS.}

Colombia, Corte Constitucional, (2006). Sentencia C- 669 de 2006, M.P. Dr. ALVARO TAFUR GALVIS, expediente D-6167, Bogotá D.C., dieciséis (16) de agosto de dos mil seis

Colombia, Corte Constitucional, (2001).Sentencia T-076 del 2001, M.P Dr. FABIO MORON DIAZ, expediente T-340 808, Bogotá D.C., enero veintinueve (29) de dos mil uno

Decreto 2090 de 2003, por el cual se definen las actividades de alto riesgo para la salud del trabajador y se modifican y señalan las condiciones, requisitos y beneficios del régimen de pensiones de los trabajadores que laboran en dichas actividades.

Decreto 1477 de 2014, por el cual se expide la Tabla de Enfermedades Laborales.

Decreto Ley 2663 de 1950 por el cual se adopta el Código Sustantivo del Trabajo.

Declaración Universal de Derechos humanos, Naciones Unidas, disponible desde internet en: http://www.un.org/es/documents/udhr/

Diccionario de la Lengua Española, Real Academia Española, edición 23, publicada en octubre de 2014. Disponible desde internet en: http://lema.rae.es/drae/?val=vacaci\%C3\%B3n+ 
Fonseca Patiño Paola, Heredia Villarroya José Y Navarrete Tarquino Diana (2010). Vigilancia Médica para los trabajadores expuestos a benceno, tolueno y xileno, articulo investigativo, Universidad del Rosario,

Henao Hidrón Javier, (2010) Constitución Política comentada, Decimoséptima edición, Editorial TEMIS S.A, Bogotá, Colombia, Pg. 38.

Lóyzaga De La Cueva Octavio F. Comentarios y reflexiones en torno a los días de descanso y vacaciones, Articulo disponible desde internet en: http://www.azc.uam.mx/publicaciones/alegatos/pdfs/72/79-09.pdf

OIT. Convenio sobre las vacaciones pagadas, 1936 (revisado), 1970, disponible desde internet en:

http://www.ilo.org/dyn/normlex/es/f?p=NORMLEXPUB:12100:0::NO::P12100_INST RUMENT_ID:312277 